\title{
Avaliação da distribuição de cirurgiões-dentistas especialistas em Dentística no Brasil
}

\author{
Evaluation of the distribution of dentistry specialists in Dentística in Brazil
}

\section{Evaluación de la distribución de odontólogos especialistas en Dentística en Brasil}

Recebido: 14/05/2020 | Revisado: 17/05/2020 | Aceito: 20/05/2020 | Publicado: 30/05/2020

André Rodrigo Justino da Silva ORCID: http://orcid.org/0000-0002-9864-292X

Universidade de Pernambuco, Brasil

E-mail: a.rodrigo2010@ hotmail.com

Andressa Nascimento de Souza

ORCID: http://orcid.org/0000-0003-3307-4893

Universidade Federal do Rio Grande do Norte, Brasil

E-mail: andressa.nasouza@gmail.com

\section{Resumo}

O objetivo é avaliar e caracterizar a distribuição no Brasil dos cirurgiões-dentistas que possuem registros no Conselho Federal de Odontologia como especialistas em Dentística ou Dentística Restauradora, fornecendo uma visão de mercado de trabalho. Realizou-se levantamento de dados baseado em pesquisa documental a partir de arquivos públicos, com caracterização quantitativa e percentual e apresentação dos resultados na forma de estatística descritiva. Dos 333.030 cirurgiõesdentistas registrados em todo o território nacional, apenas 6.363 possuem registros como especialistas em Dentística ou Dentística Restauradora, correspondente a aproximadamente 2\%. Quatro dos cinco estados com menor percentual de registros nas especialidades estudadas são pertencentes à região Norte do país, enquanto que os cinco estados brasileiros com maior percentual são das regiões Sudeste e Sul, dos quais São Paulo ocupa a primeira posição. A região Nordeste proporcionalmente se mostra como a menos competitiva e mais promissora para os especialistas em Dentística e Dentística Restauradora, ao contrário da região Centro-oeste. Conclui-se que as especialidades estudadas se mostram extremamente promissoras, visto que são essenciais para o desenvolvimento de uma odontologia de excelência, além de ser necessário se atentar às regiões e estados mais favoráveis para o desenvolvimento profissional desses especialistas, já que existem diferenças significativas quanto à distribuição espacial no país. 
Palavras-chave: Recursos Humanos em Odontologia; Dentística Operatória; Mercado de Trabalho.

\begin{abstract}
The objective is to evaluate and characterize the distribution in Brazil of dentists who have registered with the Federal Council of Dentistry as specialists in Dentística ou Dentística Restauradora, providing a view of the labor market. Data were collected based on documentary research from public archives, with quantitative and percentage characterization and presentation of results in the form of descriptive statistics. Of the 333,030 dentists registered throughout the country, only 6,363 have records as specialists in Dentística ou Dentística Restauradora, corresponding to approximately $2 \%$. Four of the five states with the lowest percentage of records in the studied specialties are from the North of the country, while the five brazilian states with the highest percentage are from the Southeast and South, with São Paulo occupying the first position. The Northeast region proportionally shows itself as the least competitive and most promising for specialists in Dentística ou Dentística Restauradora, in contrast to the Midwest region. It is concluded that the studied specialties are extremely promising, since they are essential for the development of excellent dentistry, in addition to the need to pay attention to the most favorable regions and states for the professional development of these specialists, since there are significant differences in terms of spatial distribution in the country.
\end{abstract}

Keywords: Dental Staff; Dentistry, Operative; Job Market.

\title{
Resumen
}

El objetivo es evaluar y caracterizar la distribución en Brasil de los dentistas que se han registrado en el Consejo Federal de Odontología como especialistas en Dentística ou Dentística Restauradora, brindando una visión del mercado laboral. Los datos fueron recolectados con base en investigaciones documentales de archivos públicos, con caracterización cuantitativa y porcentual y presentación de resultados en forma de estadística descriptiva. De los 333,030 odontólogos registrados en todo el país, solo 6,363 tienen registros como especialistas en Dentística ou Dentística Restauradora, lo que corresponde aproximadamente al $2 \%$. Cuatro de los cinco estados con el porcentaje más bajo de registros en las especialidades estudiadas son del norte del país, mientras que los cinco estados brasileños con el porcentaje más alto son del sudeste y sur, con São Paulo ocupando la primera posición. La región del Nordeste se muestra proporcionalmente como la menos competitiva y la más prometedora para especialistas en Dentística ou Dentística Restauradora, a diferencia de la región del Medio Oeste. Se concluye que las especialidades estudiadas son extremadamente prometedoras, ya que son esenciales para el desarrollo de una excelente odontología, además de la necesidad de prestar atención a las regiones y estados más favorables para el desarrollo profesional de estos especialistas, ya que existen diferencias significativas en términos de distribución espacial en el país. 
Palabras clave: Personal de Odontología; Operatoria Dental; Mercado de Trabalho.

\section{Introdução}

O mercado de trabalho odontológico brasileiro nos últimos anos vem passando por várias mudanças que se relacionam com a esfera econômica e com alterações no modelo de atenção em saúde bucal. As ofertas de vagas nos cursos de graduação aumentaram nos últimos tempos, houve ampliação das ofertas de trabalho no Sistema Único de Saúde (SUS) e interiorização das universidades (Mathias, Casani, Sagaz \& Lucietto, 2016).

O Conselho Federal de Odontologia (CFO) possui vinte e sete especialidades odontológicas reconhecidas, dentre as quais estão Dentística e Dentística Restauradora, cujos especialistas, segundo Brasil (1993), possuem competência para diagnóstico e prognóstico de doenças dentárias; procedimentos estéticos, preventivos e educativos; procedimentos conservadores de vitalidade pulpar e tratamento das lesões com restaurações e coroas individuais. Em 2003, a partir da Resolução CFO 22/2003, houve alterações das nomenclaturas de algumas especialidades odontológicas, entre as quais a de Dentística Restauradora, que passou a ser chamada de Dentística, porém com as mesmas atribuições e competências.

De modo geral, a competitividade no campo de trabalho para os Cirurgiões-Dentistas (CD) tem aumentado e, somado a isso, está o crescimento no número de especialistas nas mais diversas áreas. Desse modo, o profissional necessita cada vez mais se aperfeiçoar e buscar se encaixar nas novas necessidades do mercado (Costa \& Rocha, 2017).

Portanto, é necessário que os profissionais de odontologia que pretendem seguir a especialidade de Dentística ou Dentística Restauradora, ou já são especialistas nessa área, tenham uma noção clara do cenário de distribuição de cirurgiões-dentistas registrados com essa especialidade pelo Brasil, para assim terem visão de mercado de trabalho e formarem suas percepções para estabelecimento profissional em locais mais promissores para essa área.

Para isso, esse trabalho se faz necessário para explanação da distribuição espacial no território nacional dos profissionais dessa área tão importante para o exercício da odontologia. Assim, o objetivo do presente estudo é avaliar e caracterizar a distribuição no Brasil dos 
cirurgiões-dentistas que possuem registros no $\mathrm{CFO}$ como especialistas em Dentística ou Dentística Restauradora.

\section{Metodologia}

O presente trabalho consiste em um estudo de documentação indireta, com levantamento de dados baseado em pesquisa documental a partir de arquivos públicos. A metodologia adotada nesta pesquisa se baseia em metodologias já adotadas em estudos prévios (Figueirêdo Júnior, Uchôa \& Pereira, 2019; Figueirêdo Júnior \& Pereira, 2019) com pequenas alterações. Consiste desse modo, na coleta de dados do número de especialistas em Dentística ou Dentística Restauradora no Brasil, com apresentação quantitativa e percentual de acordo com a aba de estatísticas, com consulta de quantitativo personalizado de profissionais e entidades ativas, do site do conselho federal de odontologia (http://website.cfo.org.br/).

Além disso, realizou-se a consulta aos dados referentes ao número de indivíduos que compõem a população do país, bem como a demografia de cada estado através do site do Instituto Brasileiro de Geografia e Estatística (IBGE, 2010), tendo como base o último censo demográfico brasileiro, realizado entre agosto e novembro de 2010. Os acessos a todas as plataformas para obtenção dos dados se deram durante o mês de maio de 2020.

Os resultados obtidos estão apresentados na forma de estatística descritiva, com destaque para a relação proporcional, medida em nível de região, entre a taxa populacional para cada profissional especialista registrado.

\section{Resultados}

Até a data de realização da pesquisa, os dados presentes nos sites do CFO apontam o total de 333.030 cirurgiões-dentistas registrados em todo o território nacional, dos quais 6.363 possuem registros como especialistas em Dentística ou Dentística Restauradora. Os números de CDs pertencentes a essas especialidades compreendem aproximadamente apenas $2 \%$ do número total de profissionais registrados, estando distribuídos por cada estado de acordo com o exposto detalhadamente na tabela 1 . 
Research, Society and Development, v. 9, n. 7, e600974520, 2020

(CC BY 4.0) | ISSN 2525-3409 | DOI: http://dx.doi.org/10.33448/rsd-v9i7.4520

Tabela 1: Distribuição quantitativa e percentual de CDs especialistas em Dentística ou Dentística Restauradora ativos de acordo com cada estado brasileiro.

\begin{tabular}{|c|c|c|}
\hline Estado & $\begin{array}{c}\text { CDs especialistas em } \\
\text { Dentística e Dentística } \\
\text { Restauradora (n) }\end{array}$ & $\begin{array}{c}\text { CDs especialistas em } \\
\text { Dentística e Dentística } \\
\text { Restauradora (\%) }\end{array}$ \\
\hline Acre & 5 & 0,07 \\
\hline Alagoas & 40 & 0,62 \\
\hline Amapá & 12 & 0,18 \\
\hline Amazonas & 47 & 0,73 \\
\hline Bahia & 141 & 2,21 \\
\hline Ceará & 105 & 1,65 \\
\hline Distrito Federal & 419 & 6,58 \\
\hline Espírito Santo & 117 & 1,83 \\
\hline Goiás & 268 & 4,21 \\
\hline Maranhão & 65 & 1,02 \\
\hline Mato Grosso & 143 & 2,24 \\
\hline Mato Grosso do Sul & 103 & 1,61 \\
\hline Minas Gerais & 680 & 10,68 \\
\hline Pará & 136 & 2,13 \\
\hline Paraíba & 68 & 1,06 \\
\hline Paraná & 536 & 8,42 \\
\hline Pernambuco & 88 & 1,38 \\
\hline Piauí & 33 & 0,51 \\
\hline
\end{tabular}


Research, Society and Development, v. 9, n. 7, e600974520, 2020

(CC BY 4.0) | ISSN 2525-3409 | DOI: http://dx.doi.org/10.33448/rsd-v9i7.4520

\begin{tabular}{c|c|c}
\hline Rio de Janeiro & 761 & 11,95 \\
\hline Rio Grande do Norte & 62 & 0,97 \\
\hline Rio Grande do Sul & 598 & 9,39 \\
\hline Rondônia & 39 & 0,61 \\
\hline Roraima & 10 & 0,15 \\
\hline Santa Catarina & 341 & 5,35 \\
\hline São Paulo & 1.449 & 22,77 \\
\hline Sergipe & 61 & 0,95 \\
\hline Tocantins & 36 & 0,56 \\
\hline TOTAL & 6.363 & $100 \%$ \\
\hline
\end{tabular}

Fonte: Conselho Federal de Odontologia (CFO).

Observa-se nesta tabela que quatro dos cinco estados com menor percentual de registros nas especialidades estudadas neste trabalho são pertencentes à região Norte do país, sendo eles Acre, Roraima, Amapá e Tocantins. Por outro lado, de modo mais específico, o ranking dos cinco estados brasileiros com maior percentual de cirurgiões-dentistas especialistas em Dentística ou Dentística Restauradora está apresentado no gráfico 1. Sendo assim, observa-se que o estado de São Paulo (SP) se mostra como o estado com maior percentual desses especialistas, com 22,77\%; seguido do Rio de Janeiro (RJ) com 11,95\%; Minas Gerais (MG) com 10,68\%; Rio Grande do Sul (RS) com 9,39\% e Paraná (PR) com $8,42 \%$. 
Gráfico 1: Cinco estados com maior número de CDs especialistas em Dentística/Dentística Restauradora.

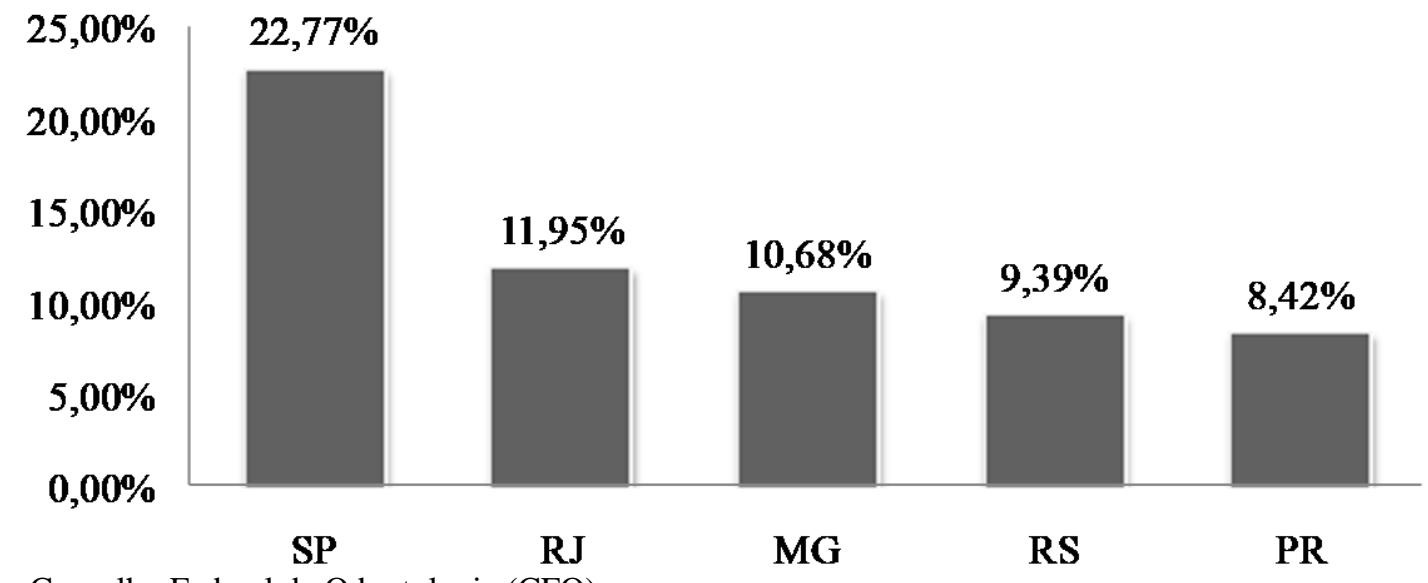

Fonte: Conselho Federal de Odontologia (CFO).

A partir deste gráfico, percebe-se que a maior concentração está nas regiões Sudeste, que ocupa as três primeiras posições, e Sul com o quarto e quinto estado. Desse modo, esses cinco estados se mostram mais saturados para as especialidades de Dentística e Dentística Restauradora em comparação com o restante do país.

Fornecendo uma visão em nível de macrorregião, está demonstrada na tabela 2 a relação proporcional entre a população de cada região brasileira e seus profissionais registrados como especialistas nas duas especialidades analisadas no presente estudo. Os dados populacionais apresentados foram obtidos a partir do último censo demográfico (2010) no site do IBGE e a relação de quantos habitantes existem para cada especialista a nível regional foi calculada a partir da junção desses dados demográficos com os dados do CFO. 
(CC BY 4.0) | ISSN 2525-3409 | DOI: http://dx.doi.org/10.33448/rsd-v9i7.4520

Tabela 2: Relação proporcional entre a população de cada região brasileira e seus profissionais registrados como especialistas em Dentística e Dentística Restauradora.

\begin{tabular}{c|c|c|c}
\hline Região & $\begin{array}{c}\text { População (censo } \\
\text { demográfico 2010) }\end{array}$ & $\begin{array}{c}\text { CDs especialistas } \\
\text { em Dentística e } \\
\text { Dentística } \\
\text { Restauradora (n) }\end{array}$ & $\begin{array}{c}\text { Relação } \\
\text { proporcional entre } \\
\text { população e CDs } \\
\text { especialistas }\end{array}$ \\
\hline Norte & 15.864 .454 & 285 & $1 / 55.664$ \\
\hline Nordeste & 53.081 .950 & 663 & $1 / 80.063$ \\
\hline Sudeste & 80.364 .410 & 3.007 & $1 / 26.725$ \\
\hline Sul & 27.386 .891 & 1.475 & $1 / 15.067$ \\
\hline Centro-oeste & 14.058 .094 & 933 & \\
\hline
\end{tabular}

Fonte: Conselho Federal de Odontologia (CFO) e Instituto Brasileiro de Geografia e Estatística (IBGE, 2010).

Verifica-se que a região Nordeste proporcionalmente se mostra como a menos competitiva e mais promissora para o estabelecimento de novos especialistas em Dentística e Dentística Restauradora em comparação com as outras regiões. Ao passo que a região Centrooeste é a que possui esses especialistas já estabelecidos em maior número em relação à população que possui, podendo assim ser considerada como a macrorregião com o mercado de trabalho mais competitivo para essas especialidades.

\section{Discussão}

O sistema de atenção em odontologia se encontra em crise, já que de um lado, tem-se a expansão acelerada de cursos de odontologia no país que leva a um estreitamento agressivo do mercado de trabalho e acaba levando os profissionais a se submeterem a práticas inadmissíveis, enquanto que por outro lado, observa-se que uma grande parcela da população permanece sem assistência necessária, aumentando a demanda e difamando, de certo modo, a profissão pela sociedade. Tudo isso em conjunto influencia na concorrência e contribui para a desunião da classe odontológica brasileira (Ferreira, Ferreira \& Freire, 2013).

A educação continuada através de cursos de especialização ajuda a tornar o profissional munido de conhecimentos científicos específicos para uma determinada área, 
aperfeiçoando suas práticas. A especialidade, segundo o $\mathrm{CFO}$ corresponde a uma área específica executada por um profissional capacitado para execução de procedimentos mais complexos (Brasil, 2005; Figueirêdo Júnior \& Pereira, 2019).

Levando em conta o fator gênero, o mercado de trabalho de CDs especialistas no Brasil é majoritariamente composto por profissionais do gênero feminino. Isso é visto em várias especialidades como na Periodontia, Ortodontia, Endodontia, bem como nas duas especialidades destacadas no presente estudo: Dentística e Dentística Restauradora (Figueirêdo Júnior \& Pereira, 2019).

Assim como no estudo de Figueirêdo Júnior \& Pereira (2019), é importante salientar que os números de profissionais registrados como especialistas em Dentística e Dentística Restauradora expostos no presente trabalho constituem um resultado da necessidade pessoal de crescimento e realização profissional, bem como o reflexo das tendências do mercado de trabalho, que exige cada vez mais a prestação de serviços odontológicos especializados.

A caracterização da distribuição de CDs especialistas pelo país e sua relação com o número de habitantes revela que em alguns estados e regiões do Brasil há uma saturação de profissionais, enquanto que outros refletem relativa carência dos mesmos (Figueirêdo Júnior, Uchôa \& Pereira, 2019), como verificado no presente estudo para as duas especialidades explanadas. Desse modo, é possível obter a partir dos resultados deste artigo as possíveis áreas ou regiões com maior potencial promissor para o mercado de trabalho dos especialistas em Dentística e Dentística Restauradora, fornecendo índices de planejamento profissional tanto para os cirurgiões-dentistas futuros quanto aos já formados.

Sendo assim, os novos profissionais, uma vez preparados e conscientes dos obstáculos que poderão encarar, encontrarão uma forma de superá-los, passando a viver uma nova fase no futuro que poderá até ser nomeada "Era da Superação em Odontologia" (Ferreira, Ferreira \& Freire, 2013).

\section{Considerações Finais}

De modo geral, observou-se que os especialistas em Dentística e Dentística Restauradora compreendem somente $2 \%$ do número total de cirurgiões-dentistas brasileiros, sendo ainda uma parcela pequena que merece mais destaque e se mostra extremamente 
promissora, visto que se trata de uma especialidade essencial para o desenvolvimento de uma odontologia de excelência. Porém é preciso se atentar às regiões e estados mais favoráveis para o desenvolvimento profissional desses especialistas, já que existem diferenças significativas quanto à distribuição espacial no país.

\section{Referências}

Brasil. Conselho Federal de Odontologia. Resolução CFO 185/1993. [Internet]. Acesso em 13 de maio de 2020, em http://www.forp.usp.br/restauradora/etica/rcfo185_93.htm\#anexos.

Brasil. Conselho Federal de Odontologia. Resolução CFO 22/2003. [Internet]. Acesso em 13 de maio de 2020, em http://www.crosp.org.br/profissionais/servicos/utilidade/especialidades/.

Brasil. Conselho Federal de Odontologia. Resolução CFO 63/2005. [Internet]. Acesso em 13 de maio de 2020, em: http://cfo.org.br/website/wp-content/uploads/2018/03/consolidacao.pdf.

Costa, D. S., Rocha, M. P. O. (2017). Cirurgião-Dentista e o mercado de trabalho no brasil: uma revisão sistemática da literatura. Id on Line Rev Mult Psic, 11(38), 102-114.

Ferreira, N. P., Ferreira, A. P., Freire, M. C. M. (2013). Mercado de trabalho na Odontologia: contextualização e perspectivas. Rev Odontol UNESP, 42(4), 304-309.

Figueirêdo Júnior, E. C., Pereira, J.V. (2019). Análise e caracterização do panorama da distribuição de Cirurgiões-Dentistas no Brasil por especialidades odontológicas. Arch Health Invest, 8(9), 465-471.

Figueirêdo Júnior, E. C., Uchôa, N. C., Pereira, J.V. (2019). Análise e caracterização do panorama da distribuição de Cirurgiões-Dentistas no Brasil. Arch Health Invest, 8(2), 63-67.

IBGE: Instituto Brasileiro de Geografia e Estatística. [Internet] 2010. Acesso em 15 de abril de 2020, em: https://cidades.ibge.gov.br/brasil/panorama.

Mathias, M. P., Casani, E., Sagaz, S. M., \& Lucietto, D. A. (2016). Oferta de cirurgiõesdentistas e faculdades no Brasil: repercussões sobre a prática odontológica. In: X Mostra de 
Iniciação Científica e Extensão Comunitária e IX Mostra de Pesquisa de Pós-Graduação da IMED 2016, 1-11.

\section{Porcentagem de contribuição de cada autor no manuscrito}

André Rodrigo Justino da Silva - 70\%

Andressa Nascimento de Souza - 30\% 